\title{
HITTING ALL MAXIMAL BICLIQUES OF A GRAPH
}

\author{
THINH D. NGUYEN
}

\begin{abstract}
Given a graph $G(V, E)$, finding a small subset of $V$ hitting all maximal bicliques of $G$ is a difficult problem. This paper shows this claim holds true.
\end{abstract}

\section{INTRODUCTION AND NOTATION}

Given a graph $G(V, E)$, a set $C \subseteq V$ is called a biclique if and only if $G[C]$ is a complete bipartite graph, where $G[C]$ is the subgraph induced by $C$. A biclique is called maximal if it is not a subset of a larger biclique. Then our problem asks whether there exists a subset of $V$ of size at most $k$ hitting all maximal bicliques of $G$, i.e., can we find $k$ vertices in $V$ such that every maximal bicliques of $G$ conntains at least one vertex of these $k$ vertices. Denote our proble by Maximal Biclique HitTing Set.

Claim 1.1. We have that Vertex Cover $\leq_{p}$ Maximal Biclique Hitting Set

In this claim, VerTex COVER denotes the well-known Vertex Cover problem. Garey and Johnson [1] proves its NP-hardness. As we shall see, the definition of a graph is of technicality ambiguity. This techinicality is addressed in the next section. And then in the subsequent section, we prove that both of the interpretations of the term maximal biclique result in hard problems.

\section{TEChNiCALity AMBiguity}

The ambiguity arises from the question "Is an independent set a biclique?".

Normally, we assume that a biclique has at least one vertex in each part (and thus at least one edge). But in many ways, it makes more sense to consider an independent set (IS) to be a biclique too: it is a complete bipartite subgraph induced by two disjoint vertex subsets $A$ and $B$, where it just happens that one of the subsets is empty. So it (vacuously) meets the criteria. (Similarly, a one-vertex graph is still considered a bipartite graph, and still considered a clique.) We will first give a reduction that considers only complete induced bipartite subgraphs with both vertex sets nonempty to be bicliques, and then describe how to modify the construction when independent sets are also considered to be bicliques (which turned out to be much trickier).

We will call the first interpretation the "edgy" interpretation and denote the corresponding problem Maximal Biclique Hitting Set - Edgy (MBHS). Such a term is getting its name from the fact that a biclique that contains at least one edge (i.e., is not an IS) is an "edgy" biclique. And the second interpretation is called

Key words and phrases. biclique, cover, graph, Hamiltonian, hitting, maximal, vertex.

Perebor. 
"non-edgy" interpretation and denote the corresponding problem MAXIMAL BICliques Hitting Set - NonEdgy. So, in order to fully established our claim ??, we split it into two claims as follows. Both claims will be proved in the next section.

Claim 2.1. We have that Vertex Cover $\leq_{p}$ Maximal Biclique Hitting Set - EDGY

Claim 2.2. We have that Vertex Cover $\leq_{p}$ Maximal Biclique Hitting Set - NONEDGY

\section{Details of the two constructions}

3.1. Proof of the claim 2.1. In this subsection, we give the reduction from Vertex Cover for "edgy" bicliques interpretation.

\section{Details of the construction for "edgy" bicliques}

The following construction builds an MBHS instance $\left(G^{\prime}=\left(V^{\prime}, E^{\prime}\right), k^{\prime}\right)$ that replaces every edge in the input graph $G=(V, E)$ with a "rectangle" in which the two pairs of diagonally opposite vertices form a 4-vertex maximal biclique.

Add all vertices and edges in $G$ to $G^{\prime}$. For every edge $u v$ in $E$, create two new vertices $x_{u v}$ and $y_{u v}$ in $G^{\prime}$, and the three edges $x_{u v} y_{u v}, u y_{u v}$ and $v x_{u v}$. Set $A_{u v}=\left\{u, x_{u v}\right\}$ and $B_{u v}=\left\{v, y_{u v}\right\}$ and observe that $\left(A_{u v}, B_{u v}\right)$ forms a biclique with 2 vertices in each part.

To see that this biclique is maximal, consider any other vertex $z \in V^{\prime} \backslash\left\{u, v, x_{u v}, y_{u v}\right\}$. To be able to add $z$ to $A_{u v}$ to form a larger biclique $\left(A_{u v}^{\prime}, B_{u v}\right), z$ must be adjacent to both $v$ and $y_{u v}$, but by construction, the only vertices adjacent to $y_{u v}$ are $x_{u v}$ and $u$, and $z$ is not either of those. Similar reasoning shows that $z$ cannot be added to the other side $\left(B_{u v}\right)$ either. Since $z$ was arbitrary, $\left(A_{u v}, B_{u v}\right)$ must be a maximal biclique.

Finally, set the threshold parameter $k^{\prime}$ for the MBHS instance to $k$. This concludes the construction. We will now show that the constructed MBHS instance is a YES instance if and only if the original VC instance is a YES instance.

\section{Proof of correctness}

Proof. (of claim 2.1)

- If there is a solution to the $\mathrm{VC}$ instance of size at most $k$, then there is a solution to the constructed MBHS instance of size at most $k^{\prime}$. The VC directly gives a solution to the MBHS instance, since every maximal biclique in $G^{\prime}$ (both the rectangle bicliques that we created, and any bicliques already present in $G$ ) contains some edge of $G$, and every edge of $G$ contains at least one vertex in the $\mathrm{VC}$ solution.

- If there is a solution to the constructed MBHS instance of size at most $k^{\prime}$, then there is a solution to the original VC instance of size at most $k$. Assume there is an edge $u v$ in $G$ whose corresponding edge in $G^{\prime}$ is not covered (i.e., neither of its endpoint vertices is in the MBHS solution), since otherwise we are already done. Let $\left(\left\{u, x_{u v}\right\},\left\{v, y_{u v}\right\}\right)$ be the unique rectangle maximal biclique that contains $u v$ in $G^{\prime}$. Then it must be that at least one of $x_{u v}$ or $y_{u v}$ is in the MBHS solution, since there is no other way to hit this biclique. Replace whichever one of these two vertices is in the solution (choose arbitrarily if both are) with either $u$ or $v$ (again, choose arbitrarily) to create a new MBHS solution of the same size (at most $k$ ) 
with one more $G$-edge covered. This can be repeated until every edge in $G$ is covered by a vertex in the MBHS solution, which remains of size at most $k$. Finally, discard any remaining "rectangle vertices" (vertices not in $V$ ) from the solution, leaving a $\mathrm{VC}$ of size at most $k$.

3.2. Proof of the claim 2.2. When independent sets are considered bicliques too, we show that the problem remains hard.

\section{Details of the construction for "non-edgy" bicliques}

If independent sets are also bicliques, the construction above can fail, most obviously on a graph containing vertices but no edges (which has a single maximal biclique, but an empty $\mathrm{VC})$.

Create $|V|+1$ copies of $G^{\prime}$ (that is, of the graph constructed for the reduction for edgy maximal bicliques). Call this new graph $G^{\prime \prime}$. The key observation is that in $G^{\prime \prime}$, **there are $|V|+1$ copies of each maximal edgy biclique in $G^{\prime}$ that all need to be hit separately, but a set of vertices that hits every maximal IS in a single copy of $G^{\prime}$ suffices to hit every maximal IS in $G^{\prime \prime}$.** (There are actually vastly more maximal ISes in $G^{\prime \prime}$ than in $G^{\prime}$, since to form a maximal IS in $G^{\prime \prime}$, we may independently choose a maximal IS from each copy of $G^{\prime}$. But an IS in $G^{\prime \prime}$ cannot be maximal unless it includes *some* $G^{\prime \prime}$-IS in each copy - so it suffices to choose a particular copy and hit every maximal IS in that copy.)

Let $k^{\prime \prime}$ be the threshold parameter that together with $G^{\prime \prime}$ forms the constructed instance of MBHS. Set $k^{\prime \prime}=k(|V|+1)+|V|$. Essentially this allows us to spend $k$ vertices hitting edgy maximal bicliques in each of the $|V|+1$ copies of $G^{\prime}$, plus up to $|V|$ vertices to hit maximal ISes.

That ends the description of the construction. We will now show that the constructed MBHS instance is a YES instance if and only if the original VC instance is a Yes instance.

\section{Proof of correctness}

Proof. (of claim 2.2)

- If there is a solution to the $\mathrm{VC}$ instance of size at most $k$, then there is a solution to the constructed MBHS instance of size at most $k^{\prime \prime}$. To see this, first replicate the VC solution across all $|V|+1$ copies of $G^{\prime}$ in $G^{\prime \prime}$ : these vertices hit every *edgy* maximal biclique for a cost of $k(|V|+1)$. Now add every remaining vertex from the first copy of $G^{\prime}$ to the solution, ensuring that we hit every maximal IS in that copy of $G^{\prime}$, and thus every maximal IS in $G^{\prime \prime}$ overall, for at most $|V|$ more vertices. Every maximal biclique is now hit and we have used at most $k(|V|+1)+|V|=k^{\prime \prime}$ vertices.

- If there is a solution to the constructed MBHS instance of size at most $k^{\prime \prime}$, then there is a solution to the original VC instance of size at most $k$. By the pigeonhole principle, at least one of the $|V|+1$ copies of $G^{\prime}$ has at most $k$ vertices in the solution. These $k$ vertices necessarily hit all edgy maximal bicliques in that copy, since there is no other way to hit these bicliques, and so can be transformed into a solution to the original VC instance of size at most $k$ in the same way as described earlier for the "edgy bicliques" version of the reduction.

Obviously all steps can be carried out in polynomial time, so the MBHS problem is NP-hard. (It's not clear that it is in NP though: there could be 
exponentially many maximal ISes (consider a $3 n$-vertex graph consisting of $n$ vertex-disjoint triangles), so checking that each one is hit takes too long. Whether MBHS is in NP is an open question.)

\section{Conclusion}

As long as we study a mathematical conjecture, we should encourage ourselves of having enough labouring hours on Prasolov and Sharygin maths books. Then, reading some articles on theory of computing like [2] is a good practice. Only after that, could we think of the ultimate final for all mathematics sciences.

\section{REFERENCES}

1. Michael R. Garey, David S. Johnson, Computers and Intractability: A Guide to the Theory of NP-Completeness

2. Phan Dinh Dieu, Le Cong Thanh, Le Tuan Hoa, Average Polyno-mial Time Complexity of Some NP-Complete Problems, Theor. Comput. Sci. 46(3): 219-237 (1986)

Current address: Department of Mathematics, Moscow State University

Email address: kosmofarmer@yandex.com 